\title{
ON INVARIANT OPERATIONS ON A MANIFOLD WITH CONNECTION OR METRIC
}

\author{
JAN SLOVÁK
}

\begin{abstract}
The study of the invariant local operations on exterior forms is a classical and well-understood subject. However, we reconsider the problem assuming only the locality of the operations and we still derive a complete explicit classification. In particular, both the finiteness of the order and polynomiality follow. Hence the present paper considerably generalizes the classical results and suggests a nice axiomatic definition of well-known operations.

First we present our main technical tools. Next we classify all (possibly nonlinear) operators on exterior forms on a manifold with connection; they are generated by the Chern forms, the exterior differential, and the wedge product. Finally, we discuss operations on Riemannian manifolds. The results involve a generalization of the well-known Gilkey theorem on the uniqueness of the Pontryagin forms.
\end{abstract}

\section{The main tools}

1.1. Bundle functors. Our aim is to classify geometrically defined operators with values in exterior forms on a manifold endowed with connection or metric. A suitable explicit formulation of the problem is provided by the general theory of bundle functors and natural operators which was worked out after Nijenhuis's paper [8] (see, e.g., Terng [12], Palais and Terng [9], and Epstein and Thurston [3]). Recently, this general and precise setting for geometric objects and operations has been developed systematically and extended to more general categories of manifolds in the monograph [5] by Kolár, Michor, and Slovák. Let us recall that a bundle functor on the category $\mathscr{M} f_{m}$ of $m$-dimensional manifolds and local diffeomorphisms (i.e., globally defined but locally invertible smooth mappings) with values in the category $\mathscr{F} \mathscr{M}$ of fibered manifolds is a functor $F: \mathscr{M} f_{m} \rightarrow \mathscr{F} \mathscr{M}$ which satisfies

(i) $B \circ F=\operatorname{id}_{\mathscr{M} f_{m}}$, where $B: \mathscr{F} \mathscr{M} \rightarrow \mathscr{M} f$ is the base functor,

Received May 2, 1991.

Key words and phrases. Invariant operators, natural operators, bundle functors, Chern forms, Pontryagin forms. 
(ii) for every inclusion $i_{U}: U \hookrightarrow M$ of an open submanifold, $F U$ is the restriction $p_{M}^{-1}(U)$ of the value $F M=\left(p_{M}: F M \rightarrow M\right)$ to $U$ and $F i_{U}$ is the inclusion $p_{M}^{-1}(U) \hookrightarrow F M$.

If for every point $x \in M$ and every local diffeomorphism $f: M \rightarrow M^{\prime}$ the restriction of $F f$ to the fiber $F_{x} M$ over $x$ depends only on the $r$-jet $j_{x}^{r} f$, then the bundle functor $F$ is said to be of order $r$.

In the sequel we shall deal with the following bundle functors: $\Lambda^{p} T^{*}-$ the bundle functor of exterior forms; $T^{(q, p)}-p$-times covariant and $q$ times contravariant tensor fields; $S_{+}^{2} T^{*}$ - the subbundle of positive definite symmetric 2-forms in $S^{2} T^{*}$, i.e., the Riemannian metrics; $P^{1}$-the first order frame bundle, i.e., $P^{1} M=\operatorname{inv} J_{0}^{1}\left(\mathbb{R}^{m}, M\right) ; Q P^{1}$-the connection bundle on $P^{1}$, i.e., $\left(Q P^{1}\right) M=J^{1} P^{1} M / G L(m, \mathbb{R})$ is the bundle of linear connections on $M$; and $Q_{\tau} P^{1}$-the subbundle in $Q P^{1}$ of symmetric linear connections. The last two bundle functors are of order two. All the other ones are first order bundle functors and moreover vector bundle functors, except $S_{+}^{2} T^{*}$ which is an open subbundle functor in $S^{2} T^{*}$.

The expression $F \times G$ means the product of two bundle functors $F$ and $G$. Since all natural transformations between bundle functors on $\mathscr{M} f_{m}$ are formed by morphisms over identities, the value $(F \times G) M$ is the fibered product $F M \times{ }_{M} G M$ and similarly for morphisms.

1.2. Natural operators. If $Y \rightarrow M$ is a fibered manifold, we write $C^{\infty}(Y)$ for the space of all smooth sections of $Y$. Given two fibered manifolds $Y$ and $Y^{\prime}$ over the base $M$, a mapping $D: C^{\infty}(Y) \rightarrow C^{\infty}\left(Y^{\prime}\right)$ is called a local smooth operator if for each point $x \in M$ and every section $s \in C^{\infty}(Y)$, the value $D s(x)$ depends only on the germ of $s$ at $x$ and if all smoothly parametrized families $s_{t}, t \in \mathbb{R}^{k}$, of sections are transformed into smoothly parametrized families.

A bundle functor $F$ defines for every $m$-dimensional manifold $M$ an action of the diffeomorphism group $\operatorname{Diff}(M)$ on the value $F M$. This induces the action on the spaces of sections $f_{*} s=F f \circ s \circ f^{-1}$. A natural operator $D: F \rightarrow E$ between two bundle functors is a system of local smooth operators $D_{M}: C^{\infty}(F M) \rightarrow C^{\infty}(E M), M \in \mathrm{Ob} \mathscr{M} f_{m}$, such that

(i) $f_{*}\left(D_{M} s\right)=D_{N}\left(f_{*} s\right)$ for all sections $s \in C^{\infty}(F M)$ and all diffeomorphisms $f: M \rightarrow N$;

(ii) $\left.\left(D_{M} s\right)\right|_{U}=D_{U}\left(\left.s\right|_{U}\right)$ for each open submanifold $U \hookrightarrow M$.

A natural operator $D$ is said to be of order $k \leq \infty$ if the operators $D_{M}$ depend on $k$-jets of the sections only.

In view of (ii), the natural operators can be equivalently considered as 
systems of mappings defined on local sections or even on the germs of local sections. We can also view the natural operators as natural transformations $D: C^{\infty} \circ F \rightarrow C^{\infty} \circ G$ satisfying the properties corresponding to (i) and (ii).

Since we want to classify geometrically defined operators on exterior forms, we should extend the definition of bundle functors to involve functors with infinite-dimensional values and we should deal with the functor $E$ associating to each manifold $M$ the infinite-dimensional space of all operators $C^{\infty}\left(\Lambda^{p} T^{*} M\right) \rightarrow C^{\infty}\left(\Lambda T^{*} M\right)$ and natural transformations $D:$ Id $\rightarrow E$ (i.e., the corresponding operators $D_{M}$ have no arguments). Further, we should replace the category $\mathscr{M} f_{m}$ by the category of Riemannian manifolds or manifolds with connection and the corresponding morphisms. However, the connections and metrics themselves are sections of values of bundle functors which are preserved by the morphisms in question, and one verifies easily that our problem is equivalent to finding all natural operators $D: Q P^{1} \times \Lambda^{p} T^{*} \rightarrow \Lambda T^{*}$ or $D: S_{+}^{2} T^{*} \times \Lambda^{p} T^{*} \rightarrow \Lambda T^{*}$ (working in the category $\mathscr{M} f_{m}$ ). We shall always restrict ourselves to symmetric linear connections, the general case can be treated by adding the torsion tensor to the arguments of the operator.

Since the objects in $\mathscr{M} f_{m}$ are locally diffeomorphic to $\mathbb{R}^{m}$ and since the action of the diffeomorphisms on $\mathbb{R}^{m}$ is transitive, every natural operator $D: F \rightarrow E$ is determined by the values of $D_{\mathbb{R}^{m}}$ on the germs of sections at the origin $0 \in \mathbb{R}^{m}$. Moreover, if $D$ is of order $k \leq \infty$ and if the order of the bundle functors $F$ and $E$ is $r$, then the whole operator is determined by the induced mapping $\mathscr{D}: J_{0}^{k}\left(F \mathbb{R}^{m}\right) \rightarrow E_{0} \mathbb{R}^{m}=J_{0}^{0}\left(E \mathbb{R}^{m}\right)$ (the so-called associated map) and the naturality implies that this mapping commutes with the induced actions of the so-called jet group $G_{m}^{r+k}=$ inv $J_{0}^{r+k}\left(\mathbb{R}^{m}, \mathbb{R}^{m}\right)_{0}$ on the fibers $J_{0}^{k}\left(F \mathbb{R}^{m}\right)$ and $E_{0} \mathbb{R}^{m}$. On the other hand, each $G_{m}^{r+k}$-equivariant mapping $J_{0}^{k}\left(F \mathbb{R}^{m}\right) \rightarrow E_{0} \mathbb{R}^{m}$ determines a unique natural operator $D: F \rightarrow E$. So we have recalled the well-known bijection between the natural operators and suitable equivariant mappings (see, e.g., Terng [12]):

Proposition. There is a bijective correspondence between the set of $k$ th order natural operators $D: F \rightarrow E$ and the set of all $G_{m}^{k+r}$-equivariant smooth mappings $J_{0}^{k}\left(F \mathbb{R}^{m}\right) \rightarrow E_{0} \mathbb{R}^{m}$.

If we deal with vector bundle functors (or affine bundle functors or subbundles of these bundle functors), the standard fibers $J_{0}^{k} F \mathbb{R}^{m}$ are vector spaces (or affine spaces or invariant submanifolds in these spaces) and we call a natural operator $D$ polynomial if the associated map $\mathscr{D}$ is 
polynomial. In this case, the coordinate expressions of the operators $D_{M}$ in arbitrary local coordinate systems on $M$ are given by a universal polynomial expression induced from $\mathscr{D}$.

1.3. Nonlinear Peetre theorem. A nonlinear generalization of Peetre's theorem on the finiteness of the order of local linear operators was proved by the author in [10]. The general result is rather technical and so we formulate a special case which we shall need.

Proposition. Let $Y \rightarrow M$ and $Y^{\prime} \rightarrow M$ be fibered manifolds and let $D: C^{\infty}(Y) \rightarrow C^{\infty}\left(Y^{\prime}\right)$ be a smooth local operator. Then for every fixed section $s \in C^{\infty}(Y)$ and for every compact set $K \subset M$, there is an order $r \in \mathbb{N}$ and a neighborhood $V$ of $s$ in the compact open $C^{\infty}$-topology such that for every $x \in K$ and $s_{1}, s_{2} \in V$ the condition $j_{x}^{r} s_{1}=j_{x}^{r} s_{2}$ implies $D s_{1}(x)=D s_{2}(x)$.

As a direct consequence of this result, we see that each natural operator $D: F \rightarrow E$ is of order $k=\infty$ and so $D$ is determined by the associated $G_{m}^{\infty}$-equivariant map $\mathscr{D}: J_{0}^{\infty}\left(F \mathbb{R}^{m}\right) \rightarrow E_{0} \mathbb{R}^{m}$.

Let us remark that a stronger version of the above proposition (without the smoothness assumption) is also proved in [5] and it is applied there in an alternative proof of the regularity and the finiteness of the order of bundle functors which avoids the original manipulation with infinitedimensional Lie groups $G_{m}^{\infty}$ (cf. [3]).

1.4. Lemma. Let $F: \mathscr{M} f_{m} \rightarrow \mathscr{F} \mathscr{M}$ be an arbitrary bundle functor and $p>q$ nonnegative integers. Then every natural operator $D: Q_{\tau} P^{1} \times$ $T^{(q, p)} \rightarrow F$ has finite order.

Proof. Let us write $E=Q_{\tau} P^{1} \times T^{(q, p)}$. By 1.3, $D$ is determined by the associated map $\mathscr{D}: J_{0}^{\infty}\left(E \mathbb{R}^{m}\right) \rightarrow F_{0} \mathbb{R}^{m}$ induced by $D_{\mathbb{R}^{m}}$. Furthermore, for every jet $j_{0}^{\infty} s \in J_{0}^{\infty}\left(E \mathbb{R}^{m}\right)$ there is an order $r<\infty$, a neighborhood $U_{r}$ of $j_{0}^{r} s$ in $J_{0}^{r}\left(E \mathbb{R}^{m}\right)$, and a smooth mapping $\mathscr{D}_{r}: U_{r} \subset J_{0}^{r}\left(E \mathbb{R}^{m}\right) \rightarrow F_{0} \mathbb{R}^{m}$ such that for all $j_{0}^{\infty} q \in V_{r}:=\left(\pi_{r}^{\infty}\right)^{-1} U_{r}$ we have $\mathscr{D}\left(j_{0}^{\infty} q\right)=\mathscr{D}_{r}\left(j_{0}^{r} q\right)$. The naturality of $D$ implies that if the open neighborhood $U_{r}$ is the maximal one with this property, then $V_{r}$ is $G_{m}^{\infty}$-invariant. The induced action of $G_{m}^{1}$ turns $J_{0}^{k}\left(E \mathbb{R}^{m}\right)$ into a sum of $G_{m}^{1}$-invariant linear subspaces in the tensor spaces $\left(\mathbb{R}^{m} \otimes \bigotimes^{l+2} \mathbb{R}^{m *}\right) \oplus\left(\otimes^{s} \mathbb{R}^{m} \otimes \bigotimes^{r+l} \mathbb{R}^{m *}\right), l \leq k$. Since $r>s$, the action of the homotheties (i.e., the center) in $G_{m}^{1}$ shows that the orbit of any neighborhood of the jet $j_{0}^{k} 0$ of the zero section under the action of $G_{m}^{\infty}$ coincides with the whole space $J_{0}^{k}\left(E \mathbb{R}^{m}\right)$.

1.5. A classical observation due probably to Schouten claims that the geometric operations of order $k$, on tensor fields depending on a connection factorize through the covariant derivatives of the arguments up to the 
order $k$, and through the curvature and its covariant derivatives up to the order $k-1$. Several authors derived more precise formulations involving some further assumptions (see, e.g., Lubczonok [7], Atiyah, Bott, and Patodi [1], Epstein [2], and Krupka and Janyška [6]). A (rather technical) verification of such reduction without any additional assumption is presented in the framework of natural operators by Kolár in [5]. The proof is based on the study of $G_{m}^{k+2}$-equivariant mappings between the standard fibers and a suitable description of the orbits under the action of the jet group. On the set-theoretical level, this is a more or less classical technical computation, but the subtle point is the smoothness.

Let $F$ be a first order bundle functor on $\mathscr{M} f_{m}$, and $E$ an open natural subbundle of a natural vector bundle $\bar{E}$ on $\mathscr{M} f_{m}$. The curvature and its covariant derivatives are natural operators $\rho_{k}: Q_{\tau} P^{1} \rightarrow R_{k}$, with values in tensor bundles $R_{k}, R_{k} \mathbb{R}^{m}=\mathbb{R}^{m} \times W_{k}, W_{0}=\mathbb{R}^{m} \otimes \mathbb{R}^{m *} \otimes \Lambda^{2} \mathbb{R}^{m *}$, $W_{k+1}=W_{k} \otimes \mathbb{R}^{m *}$. Similarly, the covariant differentiation of sections of $E$ forms natural operators $d_{k}: Q_{\tau} P^{1} \times E \rightarrow E_{k}$, where $E_{0}=\bar{E}$, $E_{0} \mathbb{R}^{m}=\mathbb{R}^{m} \times V_{0}, d_{0}$ is the inclusion, $E_{k} \mathbb{R}^{m}=\mathbb{R}^{m} \times V_{k}, V_{k+1}=V_{k} \otimes \mathbb{R}^{m *}$. Let us write $D^{k}=\left(\rho_{0}, \cdots, \rho_{k-2}, d_{0}, \cdots, d_{k}\right): Q_{\tau} P^{1} \times E \rightarrow R^{k-2} \times E^{k}$, where $R^{l}=R_{0} \times \cdots \times R_{l}$ and $E^{l}=E_{0} \times \cdots \times E_{l}$. All $D^{k}$ are natural operators.

Lemma. There are subbundle functors $Z^{k} \subset R^{k-2} \times E^{k}$ such that $D^{k}: Q_{\tau} P^{1} \times E \rightarrow Z^{k}$ and the associated maps $\mathscr{D}^{k}: J_{0}^{k-1}\left(Q_{\tau} P^{1} \mathbb{R}^{m}\right) \times$ $J_{0}^{k}\left(E \mathbb{R}^{m}\right) \rightarrow Z_{0}^{k} \mathbb{R}^{m}$ are surjective submersions for all $k$. Furthermore, for each point $z \in Z_{0}^{k} \mathbb{R}^{m}$ the preimage $\left(\mathscr{D}^{k}\right)^{-1}(z)$ forms one orbit under the action of the kernel $B_{1}^{k+1}$ of the projection $\pi_{1}^{k+1}: G_{m}^{k+1} \rightarrow G_{m}^{1}$.

1.6. Reduction theorem. Let us write $S_{i}$ for the tensor space $\mathbb{R}^{m} \otimes$ $S^{i+2} \mathbb{R}^{m *}, Q=S_{0}$ for the standard fiber of the bundle of symmetric connections, and

$$
\mathscr{S}: \mathscr{J}_{0}^{k-1}\left(\mathbb{R}^{m}, \mathscr{Q}\right)=\mathscr{J}_{0}^{\ell-1}\left(\mathscr{Q}_{\tau} \mathscr{P}^{1} \mathbb{R}^{m}\right) \rightarrow \mathscr{S}_{0} \times \cdots \times \mathscr{S}_{k-1}
$$

for the "symmetrization of the derivatives of the Christoffel symbols" (i.e., we express the jet space $J_{0}^{k-1}\left(Q_{\tau} P^{1} \mathbb{R}^{m}\right)$ as the sum of the tensor spaces corresponding to the individual degrees of derivatives and apply the symmetrization to the individual summands). A more or less classical construction leads to a polynomial mapping

$$
\begin{aligned}
\psi: & W_{0} \times \cdots \times W_{k-2} \times V_{0} \times \cdots \times V_{k} \times\left(S_{0} \times \cdots \times S_{k-1}\right) \\
& \rightarrow J_{0}^{k-1}\left(\mathbb{R}^{m}, Q\right) \times J_{0}^{k}\left(\mathbb{R}^{m}, V\right)
\end{aligned}
$$


such that $\psi \circ\left(\mathscr{D}^{k} \times \mathscr{S}\right)$ is the identity on $J_{0}^{k-1}\left(\mathbb{R}^{m}, Q\right) \times J_{0}^{k}\left(\mathbb{R}^{m}, V\right)$. Using this polynomial mapping and the above lemma, one concludes (cf. [5])

Proposition. For every natural operator $D: Q_{\tau} P^{1} \times E \rightarrow F$ which depends on $k$-jets of sections of the bundles EM and on $(k-1)$-jets of the connections, there is a unique natural transformation (i.e., a zero order natural operator) $\widetilde{D}: Z^{k} \rightarrow F$ such that $D=\widetilde{D} \circ D^{k}$. Furthermore, $D$ is polynomial if and only if $\widetilde{D}$ is polynomial.

Since the standard fiber $V_{0}$ of the bundle $E_{0} \mathbb{R}^{m}$ is embedded identically into $Z_{0}^{k} \mathbb{R}^{m}$ by $\mathscr{D}^{k}$, we can also add the following:

$\mathscr{D}$ is polynomial in all variables except those from $V_{0}$ with smooth real functions on $V_{0}$ as coefficients if and only if $\widetilde{D}$ is polynomial with smooth real functions on $V_{0}$ as coefficients.

1.7. Even if we have no estimate on the order, we can get an analogous result. Proceeding as in the proof of 1.4 , we obtain an open filtration of the whole fiber $J_{0}^{\infty}\left(\left(Q_{\tau} P^{1} \times E\right) \mathbb{R}^{m}\right)$ consisting of maximal $G_{m}^{\infty}$ invariant open subsets $V_{k}$ where the associated mapping $\mathscr{D}$ factorizes through $\mathscr{D}_{k}: \pi_{k}^{\infty}\left(V_{k}\right) \subset J_{0}^{k}\left(\left(Q_{\tau} P^{1} \times E\right) \mathbb{R}^{m}\right) \rightarrow F_{0} \mathbb{R}^{m}$. Now, we can apply the same procedure as in 1.6 to these invariant open submanifolds $\pi_{k}^{\infty}\left(V_{k}\right)$.

Let us define the functor $Z^{\infty}$ as the inverse limit of $Z^{k}, k \in \mathbb{N}$, with respect to the obvious natural transformations (projections) $p_{l}^{k}: Z^{k} \rightarrow Z^{l}$, $k>l$, and similarly $D^{\infty}: Q_{\tau} P^{1} \times E \rightarrow Z^{\infty}$.

Theorem. For every natural operator $D: Q_{\tau} P^{1} \times E \rightarrow F$ there is a unique natural transformation $\widetilde{D}: Z^{\infty} \rightarrow F$ such that $D=\widetilde{D} \circ D^{\infty}$. Furthermore, for every m-dimensional compact manifold $M$ and every section $s \in C^{\infty}\left(Q_{\tau} P^{1} M \times{ }_{M} E M\right)$, there is a finite order $k$ and a neighborhood $V$ of $s$ in the $C^{k}$-topology such that

$$
\widetilde{D}_{M} \mid\left(D^{\infty}\right)_{M}(V)=\left(\pi_{k}^{\infty}\right)^{*}\left(\widetilde{D}_{k}\right)_{M}
$$

for some

$$
\left(\widetilde{D}_{k}\right)_{M}:\left(D^{k}\right)_{M}(V) \rightarrow C^{\infty}\left(Z^{k} M\right),
$$

and

$$
D_{M}\left|V=\left(\widetilde{D}_{k}\right)_{M} \circ\left(D^{k}\right)_{M}\right| V .
$$

In other words, a natural operator $D: Q_{\tau} \times E \rightarrow F$ is determined in all coordinate charts of an arbitrary $m$-dimensional manifold $M$ by a universal smooth mapping defined on the curvatures and all their covariant 
derivatives and on the sections of $E M$ and all their covariant derivatives, which depends "locally" on a only finite number of these arguments.

1.8. The Riemannian case. On Riemannian manifolds, there is the natural operator $\Gamma: S_{+}^{2} T^{*} \rightarrow Q_{\tau} P^{1}$ defined by the Levi-Cività connection. Every operator $S_{+}^{2} T^{*} \times E \rightarrow F$ can be viewed as an operator $Q_{\tau} P^{1} \times$ $S_{+}^{2} T^{*} \times E \rightarrow F$, independent of the first argument. Since $S_{+}^{2} T^{*} \subset S^{2} T^{*}$ is an open subbundle functor, we can consider the compositions $D^{k}$ 。 $(\Gamma, \mathrm{id}): S_{+}^{2} T^{*} \times E \rightarrow Q_{\tau} P^{1} \times S_{+}^{2} T^{*} \times E \rightarrow R^{k-2} \times\left(S_{+}^{2} T^{*} \times E\right)^{k}$ and apply the above proposition. Since all covariant derivatives of the metric with respect to the metric connection are zero, the covariant derivatives of the metric will not appear in the codomain of the operators $D^{k}$. Hence we get (cf. [5])

Proposition. There are subbundle functors $Z^{k} \subset R^{k-2} \times E^{k}$ such that $D^{k} \circ(\Gamma$, id $): S_{+}^{2} T^{*} \times E \rightarrow S_{+}^{2} T^{*} \times Z^{k}$ for all $k$, and, for every $k$ th order natural operator $D: S_{+}^{2} T^{*} \times E \rightarrow F$, there is a natural transformation $\widetilde{D}: S_{+}^{2} T^{*} \times Z^{k} \rightarrow F$ such that $D=\widetilde{D} \circ D^{k} \circ(\Gamma \times \mathrm{id})$.

Let us notice that the bundles $Z^{k} M$ involve the curvature of the Riemannian connection on $M$, its covariant derivatives, and the covariant derivatives of the sections of $E M$. Similarly as above, we define the inverse limits $Z^{\infty}$ and $D^{\infty}$ and we get

Corollary. For every natural operator $D: S_{+}^{2} T^{*} \times E \rightarrow F$ there is a natural transformation $\widetilde{D}: S_{+}^{2} T^{*} \times Z^{\infty} \rightarrow F$ such that $D=\widetilde{D} \circ D^{\infty} \circ$ $(\Gamma$, id). Furthermore, for every $m$-dimensional compact manifold $M$ and every section $s \in C^{\infty}\left(S_{+}^{2} T^{*} M \times_{M} E M\right)$, there is a finite order $k$ and $a$ neighborhood $V$ of $s$ in the $C^{k}$-topology such that

$$
\widetilde{D}_{M} \mid\left(D^{\infty} \circ(\Gamma, \mathrm{id})\right)_{M}(V)=\left(\pi_{k}^{\infty}\right)^{*}\left(\widetilde{D}_{k}\right)_{M},
$$

where

$$
\begin{gathered}
\left(\widetilde{D}_{k}\right)_{M}:\left(D^{k} \circ(\Gamma, \mathrm{id})\right)_{M}(V) \rightarrow C^{\infty}\left(Z^{k} M\right), \\
D_{M}\left|V=\left(\widetilde{D}_{k}\right)_{M} \circ\left(D^{k}\right)_{M} \circ(\Gamma, \mathrm{id})_{M}\right| V .
\end{gathered}
$$

1.9. The polynomial operations on Riemannian manifolds. We call a natural operator $D: S_{+}^{2} T^{*} \times E \rightarrow F$ a polynomial operator on Riemannian manifolds if the associated map $\mathscr{D}: J_{0}^{\infty}\left(S_{+}^{2} \mathbb{R}^{m}\right) \times J_{0}^{\infty}\left(E \mathbb{R}^{m}\right) \rightarrow F_{0} \mathbb{R}^{m}$ depends polynomially on $k$-jets of sections of $E \mathbb{R}^{m}$ for some $k$.

By the nonlinear Peetre theorem, this means that for each Riemannian manifold $(M, g)$ the operator $D_{M}$ is given by a universal polynomial 
expression depending on the derivatives of the sections of $E M$, but the coefficients are functions depending on (locally finitely many) derivatives of the metric.

Let us consider now a $k$ th order operator $D$ and the natural transformation $\widetilde{D}$ corresponding to $D$ (see 1.8). In the center of normal coordinates, each metric has the canonical euclidean form and so the whole transformation $\widetilde{D}$ is determined by the restriction of the associated map $\widetilde{D}$ to $\{$ id $\} \times Z_{0}^{k} \mathbb{R}^{m}$. This restriction is polynomial if and only if $\widetilde{\mathscr{D}}$ depends polynomially on elements from $Z_{0}^{k} \mathbb{R}^{m}$, the metric $g_{i j}$, and the square root of the inverse of its determinant $\operatorname{det}\left(g_{i j}\right)$. Indeed, in order to find the transformation of coordinates which maps the euclidean metric to $g_{i j}$ we need to decompose $g_{i j}=A A^{T}$ with $A \in \mathrm{GL}(m, \mathbb{R})$. The same applies to $\mathscr{D}$ : if this $G_{m}^{\infty}$-equivariant map depends polynomially on the derivatives of the metric and the jets of sections of $E \mathbb{R}^{m}$, then the values of the metric appear in $\mathscr{D}$ polynomially through $g_{i j}$ and the square root of the inverse of its determinant $\operatorname{det}\left(g_{i j}\right)$.

Now, let us fix $g_{i j}$. Since $\Gamma$ depends polynomially on the 1-jet of the metric and the values of the inverse metric, it follows that $\widetilde{D}$ depends polynomially on the elements from $Z_{0}^{k} \mathbb{R}^{m}$ if and only if $\mathscr{D}$ depends polynomially on the derivatives of the metric $g_{i j}$ and on the jets of the sections of $E$ (with functions of $g_{i j}$ as coefficients), and this happens if and only if $\mathscr{D}$ depends polynomially on the jets of the metrics, the jets of the sections of $E$, and on the square root of the inverse of the determinant of $\left(g_{i j}\right)$.

Let us remark that such operations were introduced in [1] under the name regular operators; a reason why they should be distinguished among the polynomial operations on Riemannian manifolds is also shown in Stredder [11].

1.10. Invariant tensors. The above reduction procedure restricts our considerations to $G_{m}^{1}=\operatorname{GL}(m, \mathbb{R})$-equivariant mappings between the standard fibers. If we are able to prove that the operation in question is polynomial, we can apply the standard polarization technique which reduces the problem to the classification of all invariant tensors. It is well known that all $\mathrm{GL}(m, \mathbb{R})$-equivariant operations on tensors are linearly generated by permutation of indices, contractions, and tensor multiplication by invariant tensors, i.e., tensor products of identities on $\mathbb{R}^{m}$. In the Riemannian case, we meet polynomial dependence on all variables except the metric, with coefficients depending smoothly on the metric entry. Hence we have to restrict the equivariance to the orthogonal 
group $\mathrm{O}(m, \mathbb{R})$ and to use a description of all $\mathrm{O}(m)$-equivariant operations. Weyl's theorem implies that these operations are linearly generated by tensorizing by the metric tensor $g: \mathbb{R}^{m} \rightarrow \mathbb{R}^{m *}$ or by its inverse $\tilde{g}: \mathbb{R}^{m *} \rightarrow \mathbb{R}^{m}$, contractions, and permutations of indices.

\section{Manifolds with connection}

2.1. Let us first discuss the natural operators $D: Q_{\tau} P^{1} \times T^{(s, r)} \rightarrow T^{(q, p)}$ with $r>s$. Given a connection on $M$ and a tensor field, we can take covariant derivatives of the field and the covariant derivatives of the curvature, we can tensorize, we can apply any $\operatorname{GL}(m, \mathbb{R})$-equivariant operation, we can take linear combinations, and we can iterate these steps.

Lemma. All natural operators $Q_{\tau} P^{1} \times T^{(s, r)} \rightarrow T^{(q, p)}$ are obtained by this procedure. In particular, all of them are polynomial.

Proof. By 1.4, every such operator has some finite order $k$ and so it is determined by a smooth $G_{m}^{k+2}$-equivariant map $f=\left(f_{j_{1} \cdots j_{p}}^{i_{1} \cdots i_{q}}\right): J_{0}^{k}\left(\mathbb{R}^{m}, Q\right) \times$ $J_{0}^{k}\left(\mathbb{R}^{m}, V\right) \rightarrow S$, where $Q$ is the standard fiber of the connection bundle and $S=\otimes^{q} \mathbb{R}^{m} \otimes \otimes^{p} \mathbb{R}^{m *}$. Let us assume that we have chosen $k$ in such a way that $f$ depends on $(k-1)$-jets of the connections only. If we apply the equivariance of $f$ with respect to the transformation $x \mapsto c^{-1} x$, $c \in \mathbb{R}$ positive, from the center of $G_{m}^{1}$, we get

$$
\begin{aligned}
& c^{p-q} f_{j_{1} \cdots j_{p}}^{i_{1} \cdots i_{q}}\left(\Gamma_{i j}^{l}, \cdots, \Gamma_{i j, l_{1} \cdots l_{k-1}}^{l}, v_{j_{1} \cdots j_{r}}^{i_{1} \cdots i_{s}}, \cdots, v_{j_{1} \cdots j_{r} l_{1} \cdots l_{k}}^{i_{1} \cdots i_{s}}\right) \\
& =f_{j_{1} \cdots j_{p}}^{i_{1} \cdots i_{q}}\left(c \Gamma_{i j}^{l}, \cdots, c^{k} \Gamma_{i j, l_{1} \cdots l_{k-1}}^{l}, c^{r-s} v_{j_{1} \cdots j_{r}}^{i_{1} \cdots i_{s}}, \cdots, c^{r-s+k} v_{j_{1} \cdots j_{r} l_{1} \cdots l_{k}}^{i_{1} \cdots i_{s}}\right),
\end{aligned}
$$

where the subscripts $l_{j}$ denote the usual derivatives. By a general theorem on homogeneous functions (cf. [5]), $f_{j_{1} \cdots j_{p}}^{i_{1} \cdots i_{q}}$ must be sums of homogeneous polynomials.

Now, 1.6 and 1.2 imply that there is a unique smooth $G_{m}^{1}$-equivariant map $g$ on $Z_{0}^{k} \mathbb{R}^{m}$ which is a restriction of a polynomial map $\bar{g}=\left(g_{j_{1} \cdots j_{p}}^{i_{1} \cdots i_{q}}\right)$ : $W_{0} \times \cdots \times W_{k-2} \times V_{0} \times \cdots \times V_{k} \rightarrow S$ and satisfies $f=g \circ \mathscr{D}^{k}$. Therefore the coordinate expression of our operator is given by polynomial mappings

$$
g_{j_{1} \cdots j_{p}}^{i_{1} \cdots i_{q}}\left(R_{j k l}^{i}, \cdots, R_{j k l m_{1} \cdots m_{k-2}}^{i}, v_{j_{1} \cdots j_{r}}^{i_{1} \cdots i_{s}}, \cdots, v_{j_{1} \cdots j_{r} m_{1} \cdots m_{k}}^{i_{1} \cdots i_{s}}\right),
$$

where the subscripts $m_{j}$ denote the covariant derivatives. If we apply once more the equivariance with respect to the homotheties $c^{-1} \delta_{j}^{i} \in G_{m}^{1}$, 
we get

$$
\begin{aligned}
& c^{p-q} g_{j_{1} \cdots j_{p}}^{i_{1} \cdots i_{q}}\left(R_{j k l}^{i}, \cdots, R_{j k l m_{1} \cdots m_{k-2}}^{i}, v_{j_{1} \cdots j_{r}}^{i_{1} \cdots i_{s}}, \cdots, v_{j_{1} \cdots j_{r} m_{1} \cdots m_{k}}^{i_{1} \cdots i_{s}}\right) \\
& \quad=g_{j_{1} \cdots j_{p}}^{i_{1} \cdots i_{q}}\left(c^{2} R_{j k l}^{i}, \cdots, c^{k} R_{j k l m_{1} \cdots m_{k-2}}^{i}, c^{r-s} v_{j_{1} \cdots j_{r}}^{i_{1} \cdots i_{s}}, \ldots, c^{k+r-s} v_{j_{1} \cdots j_{r} m_{1} \cdots m_{k}}^{i_{1} \cdots i_{s}}\right) .
\end{aligned}
$$

This homogeneity implies that the $g$ 's must be sums of homogeneous polynomials of degrees $a_{l}$ and $b_{l}$ in the variables $R_{j k l m_{1} \cdots m_{l}}^{i}$ and $v_{j_{1} \cdots j_{r} m_{1} \cdots m_{l}}^{i_{1} \cdots i_{s}}$, satisfying

$$
2 a_{0}+\cdots+k a_{k-2}+(r-s) b_{0}+\cdots+(k+r-s) b_{k}=p-q .
$$

Now we consider the total polarization of each multihomogeneous component to obtain the linear mappings

$$
S^{a_{0}} W \otimes \cdots \otimes S^{a_{k-2}} W_{k-2} \otimes S^{b_{0}} V \otimes \cdots \otimes S^{b_{k}} V_{k} \rightarrow S .
$$

The description of all invariant tensors implies that the polynomials in question are linearly generated by monomials obtained by multiplying an appropriate number of variables $R_{j k l m_{1} \cdots m_{l}}^{i}, v_{j_{1} \cdots j_{r} m_{1} \cdots m_{l}}^{i_{1} \cdots i_{s}}$ and applying $\mathrm{GL}(m)$-equivariant operations. This yields the coordinate description of the statement of the lemma. q.e.d.

If $q=p$, then the polynomials must be of degree zero, and so only the $\mathrm{GL}(m)$-invariant tensors can appear. If $q-p<0$, there are no nonnegative integers solving (1) and so all natural operators in question are the zero operators only.

2.2. Now we can pass to our aim, the operators with values in exterior forms. In order to determine all natural operators $D: Q_{\tau} P^{1} \times T^{(0, r)} \rightarrow \Lambda T^{*}$ we have to consider the case $s=0$ in the above construction, contract all superscripts, and apply the alternation on all remaining subscripts at the very end.

Every $\operatorname{GL}(m, \mathbb{R})$-invariant polynomial $P$ defined on $\mathbb{R}^{m} \otimes \mathbb{R}^{m *}$ determines via the Chern-Weil construction a natural form, i.e., a natural operator of our type independent of the second argument. In particular, the homogeneous components of the invariant polynomial $\operatorname{det}\left(\mathbb{I}_{m}+A\right)$ give rise to the Chern forms $c_{q}$. The wedge product of exterior forms defines the algebra structure on the space of all operators in question.

Theorem. The algebra of all natural operators $D: Q_{\tau} P^{1} \times T^{(0, r)} \rightarrow \Lambda T^{*}$ is generated by the alternation, the exterior derivative $d$, and the Chern forms $c_{q}$. The operators which do not depend on the second argument are generated by the Chern forms only.

In particular, we see that all natural forms have even degrees. Since the exterior differential is natural, they must be closed. 
2.3. We shall need several lemmas in the proof of this result. Most of the covariant derivatives of the curvature and of the forms which are involved in the general construction from 2.1 are disabled by some of their symmetries during the final alternation. Let us first recall the antisymmetry of the curvature form, the first and the second Bianchi identity. In coordinates we have

$$
\begin{gathered}
R_{j k l}^{i}=-R_{j l k}^{i}, \\
R_{j k l}^{i}+R_{k l j}^{i}+R_{l j k}^{i}=0, \\
R_{j k l m}^{i}+R_{j l m k}^{i}+R_{j m k l}^{i}=0 .
\end{gathered}
$$

Lemma. The alternation of $R_{j k l m_{1} \cdots m_{s}}^{i}$ on any three indices among the first three or four subscripts is zero.

Proof. Since the covariant derivative commutes with the tensor operations like the permutation of indices, it suffices to discuss the variables $R_{j k l}^{i}$ and $R_{j k l m}^{i}$. By (3), the alternation on the subscripts in $R_{j k l}^{i}$ is zero and (4) yields the same for the alternation on $k, l, m$ in $R_{j k l m}^{i}$. In view of (2), it remains to discuss the alternation of $R_{j k l m}^{i}$ on $j, l, m$. (2) implies $R_{j k m l}^{i}=-R_{j m k l}^{i}$ and so we can rewrite this alternation as

$$
\begin{aligned}
& R_{j k l m}^{i}+R_{j m k l}^{i}+R_{j l m k}^{i}-R_{j l m k}^{i} \\
+ & R_{m k j l}^{i}+R_{m l k j}^{i}+R_{m j l k}^{i}-R_{m j l k}^{i} \\
+ & R_{l k m j}^{i}+R_{l j k m}^{i}+R_{l m j k}^{i}-R_{l m j k}^{i} .
\end{aligned}
$$

The first three entries of each row form a cyclic permutation and hence give zero. The same applies to the last column.

2.4. Lemma. For every tensor field $t=\left(t_{i_{1} \cdots i_{q}}\right)$, the alternation of its second covariant derivative $\nabla^{2} t=\left(t_{i_{1} \cdots i_{q} i_{q+1} i_{q+2}}\right)$ on all indices is zero.

Proof. Every linear connection $\Gamma_{j k}^{i}$ determines a connection $\Gamma$ with curvature $R$ on each vector bundle associated to the linear frame bundle. The components of $R$ are easily evaluated from $R_{j k l}^{i}$ using the action of $\mathfrak{g l}(m)$ on the tensor space in question. In our case, $\left(a_{j}^{i}\right) \in \mathrm{GL}(m)$ acts on a tensor $\omega_{i_{1} \cdots i_{q}}$ by $\left(a_{j}^{i}\right) \omega_{i_{1} \cdots i_{q}}=\tilde{a}_{i_{1}}^{j_{1}} \cdots \tilde{a}_{i_{q}}^{j_{q}} \omega_{i_{1} \cdots i_{q}}$, where the tilde denotes the components of the inverse matrix, and so given a tensor field $t$ we get the expression of the contraction $\langle R, t\rangle=-\sum_{s=1}^{q} R_{i_{s} i_{q+1} i_{q+2}}^{m} t_{i_{1} \cdots m \cdots i_{q}}$. If the connection is symmetric, then the Ricci identity yields $\operatorname{Alt}\left(\nabla^{2} t\right)=\langle R, t\rangle$, where the alternation concerns only the last two indices. Hence we can 
apply our alternation to this expression. Up to a constant multiple, we get

$$
\sum_{\sigma \in \Sigma} \operatorname{sgn} \sigma t_{i_{\sigma(1)} \cdots i_{\sigma(q+2)}}=-\sum_{s} \sum_{m} \sum_{\sigma} \operatorname{sgn} \sigma R_{i_{\sigma(s)}^{m} i_{\sigma(q+1)} i_{\sigma(q+2)}} t_{i_{\sigma(1)} \cdots m \cdots i_{\sigma(q)}} .
$$

Let us decompose this sum into summands with fixed $m, s$ and all $\sigma(j)$ with $j \neq s, j \neq q+1, j \neq q+2$. These are of the form

$$
\pm\left(\sum_{\bar{\sigma} \in \Sigma_{3}} \operatorname{sgn} \bar{\sigma} R_{i_{\bar{\sigma}(s)}^{m} i_{\bar{\sigma}(q+1)} i_{\bar{\sigma}(q+2)}}\right) t_{i_{\sigma(1)} \cdots m \cdots i_{\sigma(q)}} .
$$

Now the first Bianchi identity implies that all these summands vanish.

2.5. Lemma. For every tensor $t=\left(t_{i_{1} \cdots i_{q}}\right)$, the alternation of the first covariant derivative $\nabla t$ coincides with the exterior differential $d(\operatorname{Alt}(t))$.

Proof. Whenever the coordinate expressions of two natural operators coincide in one coordinate chart, the operators are equal. The first covariant derivative is of order zero in the connection argument, and at a fixed point the Christoffel symbols are zero in a suitable coordinate system. But then the formula for the alternation of the covariant derivative of the tensor $t$ coincides with that for the exterior differential of the alternated tensor at this point.

2.6. Proof of Theorem 2.2. Let us continue the discussion from 2.2 and consider first a monomial in $R$ 's and $v$ 's containing at least one quantity $R_{j k l m_{1} \cdots m_{s}}^{i}$ with $s>0$. Then there exists one term among the $R$ 's in the product with three free subscripts among the first four ones, or one term $R_{j k l}^{i}$ with all free subscripts, so that the monomial vanishes after alternation. Further, (2) and (3) imply $R_{j k l}^{i}-R_{l k j}^{i}=-R_{k l j}^{i}$. Hence we can restrict ourselves to contractions on the first two subscripts in the $R$ 's. Obviously, no subscript in the $v$ 's can be contracted since otherwise the alternation would kill one of the $R$ 's. So in view of Lemma 2.4, only the first order covariant derivatives can appear, and they yield the exterior derivatives of the alternated tensor $v$ by Lemma 2.5. Hence all the possible operators are generated by the expressions $R_{k_{1} a b}^{k_{q} a} R_{k_{2} c d}^{k_{1}} \cdots R_{k_{q} e f}^{k_{q-1}}$, where the indices $a, \cdots, f$ remain free for the alternation, $v_{i_{1} \cdots i_{r}}$ and $\operatorname{Alt}\left(v_{i_{1} \cdots i_{i} i_{r+1}}\right)$. This is a coordinate expression of the theorem.

2.7. Operations on functions. Up to now, we have assumed $r>s \geq$ 0 , so that the case $r=0$ was excluded. In this case we cannot use 1.4 and so we must apply Theorem 1.7 instead of 1.6 , but the codomain of the operations in question will still ensure the polynomiality of the operations. By 1.7 , each jet $\left(j_{0}^{\infty} \Gamma, j_{0}^{\infty} v\right)$ lies in some $G_{m}^{\infty}$-invariant open subset $V_{k} \subset J_{0}^{\infty}\left(Q_{\tau} P^{1} \mathbb{R}^{m} \times \mathbb{R}\right)$ (in the inverse limit topology) such that the 
associated mapping $\mathscr{D}$ of the operator is determined by a (locally defined) $G_{m}^{k+2}$-equivariant mapping $f: J_{0}^{k}\left(\mathbb{R}^{m}, Q\right) \times J_{0}^{k}\left(\mathbb{R}^{m}, \mathbb{R}\right) \rightarrow S$. Taking $k$ large enough, we can assume that the jet of the zero section lies in $V_{k}$. Now, proceeding as in 1.4 and 2.1 we get for every positive $c \in \mathbb{R}$ the homogeneity condition

$$
\begin{aligned}
c^{p-q} & f_{j_{1} \cdots j_{p}}^{i_{1} \cdots i_{q}}\left(\Gamma_{i j}^{l}, \cdots, \Gamma_{i j, l_{1} \cdots l_{k-1}}^{l}, v, \cdots, v_{l_{1} \cdots l_{k}}\right) \\
& =f_{j_{1} \cdots j_{p}}^{i_{1} \cdots i_{q}}\left(c \Gamma_{i j}^{l}, \cdots, c^{k} \Gamma_{i j, l_{1} \cdots l_{k-1}}^{l}, v, \cdots, c^{k} v_{l_{1} \cdots l_{k}}\right) .
\end{aligned}
$$

Thus, $f$ is a polynomial mapping in all variables except $v$ with functions of $v$ as coefficients.

Using 1.6 and 1.7 , we pass to $G_{m}^{1}$-equivariant mappings

with the homogeneity

$$
g_{j_{1} \cdots j_{p}}^{i_{1} \cdots i_{q}}\left(R_{j k l}^{i}, \cdots, R_{j k l m_{1} \cdots m_{k-2}}^{i}, v, \cdots, v_{m_{1} \cdots m_{k}}\right)
$$

$$
\begin{aligned}
c^{p-q} & g_{j_{1} \cdots j_{p}}^{i_{1} \cdots i_{q}}\left(R_{j k l}^{i}, \cdots, R_{j k l m_{1} \cdots m_{k-2}}^{i}, v, \cdots, v_{m_{1} \cdots m_{k}}\right) \\
& =g_{j_{1} \cdots j_{q}}^{i_{1} \cdots i_{p}}\left(c^{2} R_{j k l}^{i}, \cdots, c^{k} R_{j k l m_{1} \cdots m_{k-2}}^{i}, v, \cdots, c^{k} v_{m_{1} \cdots m_{k}}\right) .
\end{aligned}
$$

Hence $g$ is polynomial with smooth functions in one real variable $v$ as coefficients and the degrees of its monomials satisfy (1) with $r=s=0$. Now we can repeat the arguments from the end of 2.1 and we get

Lemma. All natural operators $D: Q_{\tau} P^{1} \times T^{(0,0)} \rightarrow T^{(q, p)}$ are obtained by iterating the following steps. Given a function, we can compose the function with an arbitrary smooth function of one real variable, we can take covariant derivatives of the function and the covariant derivatives of the curvature, we can tensorize, we can apply any $\mathrm{GL}(m, \mathbb{R})$-equivariant operation, and we can take linear combinations.

The arguments from the proof of 2.2 are also valid now and so we can extend this theorem to the case of functions.

Theorem. The algebra of all natural operators $D: Q_{\tau} P^{1} \times T^{(0,0)} \rightarrow \Lambda T^{*}$ is generated by the compositions with arbitrary smooth functions of one real variable, the exterior derivative $d$, and the Chern forms $c_{q}$.

\section{The Riemannian case}

3.1. There are many natural operators on Riemannian manifolds. In particular, using the inverse metric we can contract on any couple of indices and the complete contractions of suitable covariant derivatives of the curvature of the Levi-Cività connection give rise to natural functions of all even orders greater then one. Composing $k$ natural functions with 
any fixed smooth function $\mathbb{R}^{k} \rightarrow \mathbb{R}$, we get a new natural function. Since every natural form can be multiplied by any natural function, we see that there is no hope of describing at least all natural forms in a way similar to the above characterization of the Chern forms. However, in Riemannian geometry we meet operations with a sort of homogeneity with respect to the change of the scale of the metric and these can be described in more detail.

Definition. Let $E$ and $F$ be natural bundles over $m$-dimensional manifolds. We say that a natural operator $D: S_{+}^{2} T^{*} \times E \rightarrow F$ is conformal if $D\left(c^{2} g, s\right)=D(g, s)$ for all metrics $g$, sections $s$, and all positive $c \in \mathbb{R}$. If $F$ is a natural vector bundle and $D$ satisfies $D\left(c^{2} g, s\right)=$ $c^{\lambda} D(g, s)$, then $D$ is said to be homogeneous with weight $\lambda$.

Let us recall that the weight of the metric $g_{i j}$ is 2 (we consider the inclusion $g: S_{+}^{2} T^{*} \rightarrow S^{2} T^{*}$ ), that of its inverse $g^{i j}$ is -2 , while the curvature and all its covariant derivatives are conformal. The regular operators on Riemannian manifolds (cf. 1.9) homogeneous in the weight were studied extensively (see, e.g., Atiyah, Bott, and Patodi [1], Epstein [2], and Stredder [11]). Using the above approach, we shall recover and generalize some of their results.

Surprisingly enough we shall prove that among the homogeneous natural operators $D: S_{+}^{2} T^{*} \times T^{(0, r)} \rightarrow \Lambda T^{*}$ with nonnegative weights, there are no other ones than those obtained by evaluation of the operators from Theorem 2.2 using the Levi-Cività connection. Since the Riemannian connections have one more symmetry, namely

$$
R_{j k l}^{i}=-R_{i k l}^{j}
$$

the evaluation of the Chern forms using the Levi-Cività connection yields zero in degrees not divisible by four and the Pontryagin forms in degrees $4 l$.

3.2. Theorem. There are no nonzero homogeneous natural operators $D: S_{+}^{2} T^{*} \times T^{(0, r)} \rightarrow \Lambda T^{*}$ with a positive weight. The algebra of all conformal natural operators $D: S_{+}^{2} T^{*} \times T^{(0, r)} \rightarrow \Lambda T^{*}$ is generated by the Pontryagin forms $p_{q}$, the alternation, and the exterior differential. The operators which do not depend on the second argument are generated by the Pontryagin forms.

This generalizes the famous Gilkey theorem on the uniqueness of the Pontryagin forms (see Gilkey [4] and Atiyah, Bott, Patodi [1]). The Gilkey theorem describes the regular conformal natural forms, while we use no assumptions on the order or polynomiality or regularity, only the smoothness. 
3.3. We shall start the proof by the discussion on the natural operators $D: S_{+}^{2} T^{*} \times T^{(s, r)} \rightarrow T^{(q, p)}$ with $s<r$. Similarly to 2.7 , we use 1.7 to find $G_{m}^{\infty}$-invariant open subsets $V_{k}$ in $J_{0}^{\infty}\left(\left(S_{+}^{2} T^{*} \times T^{(s, r)}\right) \mathbb{R}^{m}\right)$ forming a filtration of the whole jet space. On these subsets $\mathscr{D}$ factorizes through smooth $G_{m}^{k+1}$-equivariant mappings

$$
f_{j_{1} \cdots j_{p}}^{i_{1} \cdots i_{q}}=f_{j_{1} \cdots j_{p}}^{i_{1} \cdots i_{q}}\left(g_{i j}, \cdots, g_{i j l_{1} \cdots l_{k}}, v_{j_{1} \cdots j_{r}}^{i_{1} \cdots i_{s}}, \cdots, v_{j_{1} \cdots j_{r} l_{1} \cdots i_{k}}^{i_{1} \cdots i_{s}}\right)
$$

defined on $\pi_{k}^{\infty} V_{k}$. Using the action of the homotheties $c^{-1} \delta_{j}^{i}$ for large $k$ 's, we get

$$
\begin{aligned}
& c^{p-q} f_{j_{1} \cdots j_{p}}^{i_{1} \cdots i_{q}}\left(g_{i j}, \cdots, g_{i j l_{1} \cdots l_{k}}, v_{j_{1} \cdots j_{r}}^{i_{1} \cdots i_{s}}, \cdots, v_{j_{1} \cdots j_{s} l_{1} \cdots l_{k}}^{i_{1} \cdots i_{i}}\right) \\
& \quad=f_{j_{1} \cdots j_{p}}^{i_{1} \cdots i_{q}}\left(c^{2} g_{i j}, \cdots, c^{2+k} g_{i j l_{1} \cdots l_{k}}, c^{r-s} v_{j_{1} \cdots j_{r}}^{i_{1} \cdots i_{s}}, \cdots, c^{r-s+k} v_{j_{1} \cdots j_{r} l_{1} \cdots l_{k}}^{i_{1} \cdots i_{s}}\right)
\end{aligned}
$$

Now, let us add the assumption that $D$ is homogeneous with weight $\lambda$, choose the change $g \mapsto c^{-1} g$ of the scale of the metric, and insert this new metric into (6). We get

$$
\begin{aligned}
& c^{p-q-\lambda} f_{j_{1} \cdots j_{p}}^{i_{1} \cdots i_{q}}\left(g_{i j}, \cdots, g_{i j l_{1} \cdots l_{k}}, v_{j_{1} \cdots j_{r}}^{i_{1} \cdots i_{s}}, \cdots, v_{j_{1} \cdots j_{r} l_{1} \cdots l_{k}}^{i_{1} \cdots i_{s}}\right) \\
& =f_{j_{1} \cdots j_{p}}^{i_{1} \cdots i_{q}}\left(g_{i j}, \cdots, c^{k} g_{i j l_{1} \cdots l_{k}}, c^{r-s} v_{j_{1} \cdots j_{r}}^{i_{1} \cdots i_{s}}, \cdots, c^{r-s+k} v_{j_{1} \cdots j_{r} l_{1} \cdots l_{k}}^{i_{1} \cdots i_{s}}\right) .
\end{aligned}
$$

This formula shows that the mappings $f_{j_{1} \cdots j_{p}}^{i_{1} \cdots i_{q}}$ are polynomials in all variables except $g_{i j}$ with functions in $g_{i j}$ as coefficients.

According to 1.8 and 1.9 , the map $\mathscr{D}$ is determined on $V_{k}$ by a polynomial mapping

$$
\omega=\left(\omega_{j_{1} \cdots j_{p}}^{i_{1} \cdots i_{q}}\left(g_{i j}, W_{j k l}^{i}, \cdots, W_{j k l m_{1} \cdots m_{k-2}}^{i}, v_{j_{1} \cdots j_{r}}^{i_{1} \cdots i_{s}}, \cdots, v_{j_{1} \cdots j_{r} m_{1} \cdots m_{k}}^{i_{1} \cdots i_{s}}\right)\right),
$$

which is $G_{m}^{1}$-equivariant on the values of the covariant derivatives of the curvatures and the sections. If we apply once more the equivariance with respect to the homothety $x \mapsto c^{-1} x$ and at the same time the change of the scale of the metric $g \mapsto c^{-1} g$, we get

$$
\begin{aligned}
c^{p-q-\lambda} \omega_{j_{1} \cdots j_{p}}^{i_{1} \cdots i_{q}}\left(g_{i j}, R_{j k l}^{i}, \cdots, R_{j k l m_{1} \cdots m_{k-2}}^{i}, v_{j_{1} \cdots j_{r}}^{i_{1} \cdots i_{s}}, \cdots, v_{j_{1} \cdots j_{r} m_{1} \cdots m_{k}}^{i_{1} \cdots i_{s}}\right) \\
=\omega_{j_{1} \cdots j_{p}}^{i_{1} \cdots i_{q}}\left(g_{i j}, c^{2} R_{j k l}^{i}, \cdots, c^{k} R_{j k l m_{1} \cdots m_{k-2}}^{i}, c^{r-s} v_{j_{1} \cdots j_{s}}^{i_{1} \cdots i_{s}}, \cdots,\right. \\
\left.c^{r-s+k} v_{j_{1} \cdots j_{r} m_{1} \cdots m_{k}}^{i_{1} \cdots i_{s}}\right) .
\end{aligned}
$$

This homogeneity shows that the polynomial functions $\omega_{j_{1} \cdots j_{p}}^{i_{1} \cdots i_{q}}$ must be sums of homogeneous polynomials with degrees $a_{l}$ and $b_{l}$ in the variables $R_{j k l m_{1} \cdots m_{l}}^{i}$ and $v_{j_{1} \cdots j_{r} m_{1} \cdots m_{l}}^{i_{1} \cdots i_{s}}$ satisfying 


$$
2 a_{0}+\cdots+k a_{k-2}+(r-s) b_{0}+\cdots+(k+r-s) b_{k}=p-q-\lambda,
$$

and their coefficients are functions depending on the $g_{i j}$ 's (in fact polynomials depending on $g_{i j}$ and on the square root of the inverse of the determinant of $g_{i j}$, cf. 1.9).

Now, we shall fix $g_{i j}=\delta_{i j}$ and use the $\mathrm{O}(m)$-equivariance of the homogeneous components of the polynomial mapping $\omega$. For this reason, we shall switch to the variables $R_{i j k l m_{1} \cdots m_{s}}=g_{i a} R_{j k l m_{1} \cdots m_{s}}^{a}$ (the $v$ 's remain). Using the standard polarization technique and the Weyl's theorem as mentioned in 1.10 , we get that each multihomogeneous component in question results from multiplication of variables $R_{i j k l m_{1} \ldots m_{s}}, v_{j_{1} \cdots j_{r} m_{1} \cdots m_{s}}^{i_{1} \cdots i_{s}}$, $s=0,1, \cdots, r$, and application of some $\mathrm{O}(m)$-equivariant tensor operations on the target space. Hence our operators result from a finite number of the following steps:

(a) take the tensor product of arbitrary covariant derivatives of the curvature tensor or the covariant derivatives of the tensor fields form the domain,

(b) tensorize by the metric or by its inverse,

(c) apply arbitrary $\mathrm{GL}(m)$-equivariant operations,

(d) take linear combinations.

3.4. If the codomain of the operator is $\Lambda T^{*}$, then all indices which were not contracted must be alternated at the end of the above procedure. Since the metric is a symmetric tensor, we get zero whenever using step (b) above and alternating on both indices. But contracting over any of them has no proper effect, for $\delta_{i j} t_{j j_{2}, \cdots, j_{s}}=t_{i j_{2}, \cdots, j_{s}}$. So we can omit step (b).

Since the Riemannian connections satisfy $R_{i j k l}=R_{k l i j}$, Lemma 2.3 and (5) yield

Lemma. The alternation of $R_{i j k l m_{1} \cdots m_{s}}$ on three arbitrary indices among the first four or five ones is zero.

Consider a monomial $P$ with degrees $a_{s}$ in $R_{i j k l m_{1} \cdots m_{s}}$ and $b_{s}$ in $v_{i_{1} \cdots i_{r} m_{1} \cdots m_{s}}$. In view of the above lemma, if $P$ remains nonzero after all alternations, then we must contract on at least two indices in each $R_{i j k l m_{1} \cdots m_{s}}$ with $s>0$ and so we can alternate on at most $2 a_{0}+\cdots+$ $k a_{k-2}+r b_{0}+\cdots+(r+k) b_{k}$ indices. This means $p \leq 2 a_{0}+\cdots+k a_{k-2}+$ $r b_{0}+\cdots+(r+k) b_{k}=p-\lambda$. Consequently $\lambda \leq 0$ if there is a nonzero natural form with weight $\lambda$. This proves the first assertion of the theorem.

Let $\lambda=0$. Since the weight of $g^{i j}$ is -2 , any contraction on two indices in the monomial decreases the weight of the operator by 2 . Every covariant derivative $R_{i j k l m_{1} \cdots m_{s}}$ of the curvature has weight 2 . So we 
must contract on exactly two indices in each $R_{i j k l m_{1} \cdots m_{s}}$, which implies that there are $s+2$ of them under alternation. But then there must appear three alternated indices among the first five if $s \neq 0$. This proves $a_{1}=$ $\cdots=a_{k}=0$. Moreover, there is no further contraction at our disposal, and so any covariant derivative of the tensors of order greater then one kills the whole monomial after alternation. Hence all the natural operators are generated by the forms $p_{q}$, the alternation, and the exterior differential. This completes the proof of the theorem.

3.5. Remark. The discussion from the proof of Theorem 3.2 can be continued for any fixed negative weight. In particular, the situation is interesting for $\lambda=-2$. Besides the well-known codifferential $\delta: \Lambda^{p} \rightarrow$ $\Lambda^{p-1}$, the compositions $d \circ \delta$ and $\delta \circ d$ (the Laplace-Beltrami operator is $\Delta=\delta \circ d+d \circ \delta$ ), and the multiplication by the scalar curvature, there appear some other simple operators. The linear operators $D: \Lambda^{p} T^{*} \rightarrow$ $\Lambda^{p} T^{*}$ were described in [11].

3.6. Exactly in the same way as in 2.7 , we can modify the proof of Theorem 3.2 for the case $r=0$. In the implicit description of all operators $D: S_{+}^{2} T^{*} \times T^{(0,0)} \rightarrow T^{(q, p)}$ in 3.3 , we have to add the compositions with smooth real functions and we get

Theorem. There are no nonzero homogeneous natural operators $D$ : $S_{+}^{2} T^{*} \times T^{(0,0)} \rightarrow \Lambda T^{*}$ with a positive weight. The algebra of all conformal natural operators $D: S_{+}^{2} T^{*} \times T^{(0,0)} \rightarrow \Lambda T^{*}$ is generated by the Pontryagin forms $p_{q}$, the compositions with arbitrary smooth functions of one real variable, and the exterior differential.

Added in proof. During his lecture series on related topics at the University of Vienna in the fall term of $1991 / 92$, the author noticed that all the results of $\S 3$ extend to pseudo-Riemannian manifolds with no additional work. The point is to rewrite (5) as $R_{i j k l}=R_{j i k l}$. Moreover, it is not difficult to include the description of natural operators on oriented pseudo-Riemannian manifolds, see pp. 29-45 in the scriptum Invariant operators on conformal manifolds, University of Vienna, 1992, 127 pp., or the forthcoming short paper On invariant operators on pseudo-Riemannian manifolds, to appear in Comment. Math. Univ. Carolinae 33 (1992), 8 pp., by the author.

\section{References}

[1] M. Atiyah, R. Bott \& V. K. Patodi, On the heat equation and the index theorem, Invent. Math. 19 (1973), 279-330.

[2] D. B. A. Epstein, Natural tensors on Riemannian manifolds, J. Differential Geometry 10 (1975), 631-645. 
[3] D. B. A. Epstein \& W. P. Thurston, Transformation groups and natural bundles, Proc. London Math. Soc. 38 (1979) 219-236.

[4] P. B. Gilkey, Curvature and the eigenvalues of the Laplacian for elliptic complexes, Advances in Math. 10 (1973) 344-382.

[5] I. Kolář, P. W. Michor \& J. Slovák, Natural operations in differential geometry, Springer, Berlin, 1992, to appear.

[6] D. Krupka \& J. Janyška, Lectures on differential invariants, Univerzita J. E. Purkyně, Brno, 1990.

[7] G. Lubczonok, On the reduction theorems, Ann. Polon. Math. 26 (1972) 125-133.

[8] A. Nijenhuis, Natural bundles and their general properties, Differential Geometry in Honor of K. Yano, Kinokuniya, Tokyo, 1972.

[9] R. S. Palais \& C. L. Terng, Natural bundles have finite order, Topology 16 (1977) 271-277.

[10] J. Slovák, Peetre theorem for nonlinear operators, Ann. Global Anal. Geom. 6 (1988) 273-283.

[11] P. Stredder, Natural differential operators on Riemannian manifolds and representations of the orthogonal and special orthogonal group, J. Differential Geometry 10 (1975) 647-660.

[12] C. L. Terng, Natural vector bundles and natural differential operators, Amer. J. Math. 100 (1978) 775-828.

Czechoslovak Academy of Science 